\title{
INFLUENCE OF ACIDOGENIC SALTS AND SODIUM BICARBONATE AS FEEDING COMPONENTS ON URINE COMPOSITION
}

\author{
C.-C. Gelfert, S. Bender and R. Staufenbiel \\ Klinik für Klauentiere, Freie Universität Berlin, D-14163 Berlin \\ Mail: c_c_gelfert@yahoo.de
}

\begin{abstract}
Rumen acidosis and rumen alkalosis are among the most frequent diseases caused by a faulty diet in dairy cattle husbandry. The economic losses are caused more by subclinical or chronic cases than by acute acidosis. Recent research has shown that sampling of urine is the best way to diagnose disturbances in the acid-base equilibrium. Acidogenic salts and sodium bicarbonate are additives often used in dairy cattle feed rations in order to prevent both parturient paresis as well as rumen acidosis. Since they interfere with the acid-base equilibrium, they may also change the urine composition which complicates diagnosis. The purpose of the following studies was to investigate the influence of both feeding additives on urine composition.
\end{abstract}

The studies took place on eight high producing dairy cattle farms in East Germany. The milk production ranged from 70001 (FCM) to 110001 /FCM), the herd size ranged from 150 to 1300 cattle. The farms were visited once a month. On these visits urine samples were taken from ten apparent healthy cows during each of five different stages of lactation The urine samples were pooled from each group and stored at $-20^{\circ} \mathrm{C}$ until laboratory testing. The concentrations of the following parameters were measured in the pooled samples: $\mathrm{pH}$, Netto-acid-base-excretion (NABE), Acids, Bases, Ammonium $\left(\mathrm{NH}_{4}^{+}\right.$), Sodium, Potassium, Chloride, Calcium, Phosphorus.

The mean $\mathrm{pH}$ for all groups showed a basic response within or above the physiological ranges. Only the cows sampled during the transit period fed acidogenic salts had $\mathrm{pH}$-values below the physiological ranges on certain times. The NABE showed an acidic response in the majority of the samples of group 2. Dependent on the quantity of the acidogenic salts feed the NABE could reach values below zero. The cows fed sodium bicarbonate had higher NABE than the animals tested during the same stage of lactation without this feed additive. The concentrations of acids showed only little variation among the nine groups. The excretion of bases in group 2 was reduced compared to group 6 and had increased in groups 3, 4 and 5 compared to groups 7, 8 and 9. Among the six groups post partum, there were no differences in the concentrations of calcium in the urine. Groups 1 and 6 showed a slightly increased excretion of calcium with concentrations around $1,5 \mathrm{mmol} / \mathrm{l}$. Cows of group $2 \mathrm{had}$ the highest excretion of calcium and the measured concentrations had been above the physiological ranges. The cows fed acidogenic salts (group 2) had the highest mean concentration of magnesium with a mean concentration above the physiological ranges. The other groups showed mean concentrations mostly within the normal ranges. The mean concentrations of potassium were above the normal ranges for the three groups ante partum. The six groups post partum had mean concentrations within the normal ranges. The cows fed sodium bicarbonate had higher concentrations than the corresponding animals of the groups without sodium bicarbonate. The cows fed sodium bicarbonate had the lowest mean concentration of chloride reaching the inferior physiological range.

Acidogenic salts and sodium bicarbonate have an influence on the composition of urine causing changes in acids, bases and major elements that is not to neglect. 
Feeding acidogenic salts produces alkaline urine. Concentrations of bases decrease, which results in a low value of NABE. The concentrations of calcium clearly increase.

Sodium bicarbonate produces basic urine. The concentration of bases and the NSBA increase, chloride concentrations decrease. For there are no signs of acidosis the decrease of $\mathrm{NH}^{+}$and phosphorus are difficult to interpret.

\title{
INTERACTIONS OF THE RUMEN WITH L-CARNITINE WHEN USED AS FEED SUPPLEMENT
}

\author{
J. Harmeyer and H.-P. Sporleder \\ Department of Physiology, School of Veterinary Medicine, Hannover, Germany
}

\begin{abstract}
Metabolic effects of supplemental L-carnitine have been reported in dairy cows (1), growing steers (2) and growing sheep (3). It was of interest to investigate whether there is an interaction between the rumen and the supplemental carnitine. Three distinct questions were asked in this context.

Is there any carnitine present in the rumen of non-supplemented ruminants?

Will carnitine be absorbed by the rumen epithelium?

Will carnitine be degraded by rumen microbes?
\end{abstract}

\section{Methods}

The experiments were carried out with six fistulated sheep, 6 to 7 years old, weighing 59 to $68 \mathrm{~kg}$. They were fed a pure hay diet ad lib.. Water was available at all times. A bolus of $1 \mathrm{~g}$ L-carnitine in the form of Carniking ${ }^{\circledR}$ was administered for 14 days immediately after the morning feed either directly into the rumen or into the omasum. CrEDTA was used as a rumen fluid marker. Artificial rumen fluid with CrEDTA and with and without L-carnitine was incubated for $3 \mathrm{~h}$ in the emptied and washed rumen. Serial samples of rumen fluid and blood were collected over 10 to $12 \mathrm{~h}$.

\section{Results}

1. L-carnitine was present in normal rumen content at concentrations of 4 to $4 \mu \mathrm{mol} / 1$. The concentration was unaffected by the time of feeding and was only 5 to $10 \%$ of that present in blood plasma. A small but significant amount of L-carnitine was secreted into the emptied and washed rumen at a rate of 15 to $30 \mu \mathrm{mol} / \mathrm{h}$ which amounted to $0.1 \%$ of the carnitine pool of the body in three hours.

2. L-Carnitine was not absorbed from the isolated and emptied, washed rumen. Three hours of incubation of artificial rumen fluid which contained $500 \mu \mathrm{mol} / \mathrm{l}$ of L-carnitine (10 times higher than blood plasma) resulted in no change in the amount of carnitine in the bathing solution and had no effect on the concentrations of free and total carnitine in blood plasma. Introduction of $1 \mathrm{~g}$ of carnitine directly into the omasum for 14 days resulted in a throughout and significantly 20 to $30 \%$ higher concentration of total carnitine in plasma than administration of this amount of carnitine into the rumen.

3. L-carnitine was extensively degraded in the rumen. When L-carnitine was administered into the rumen together with the rumen fluid marker CrEDTA L-carnitine disappeared about six times faster 
from the rumen fluid than CrEDTA. The corresponding rate constants of disappearance were 60.2 and $10.5 \% / \mathrm{h}$, respectively. Since carnitine was not absorbed from the rumen, from this it can be concluded that only $17 \%$ of the administered carnitine passed out of the rumen with the rumen fluid. The remaining fraction was probably degraded.

Summary and conclusions. L-Carnitine is not absorbed by the rumen epithelium. Instead, there is a small but significant secretion of carnitine across the epithelium into the rumen. L-carnitine is extensively degraded in the rumen. Its rate of disappearance from the rumen is about six times faster than that of CrEDTA. When L-carnitine is used as a feed supplement for ruminants carnitine should either be protected from being degraded in the rumen or losses through microbial degradation should be taken into account.

\title{
Literature
}

1. LaCount, D W; Ruppert, L D; Drackley J K. Ruminal degradation and dose response of dairy cows to dietary L-carnitine. J Dairy Sci (1996) 79, 260-269

2. Greenwood, R H; Tigtmeyer, E C; Stokka, G L; Drouillard, J S; Loest, C. Effects of L-carnitine on nitrogen retention and blood metabolites of growing steers and performance of finishing steers. J Anim Sci (2001) 79, 254-260

3. Chapa, A M; Fernandez, J M; White, T M; Bunting, L D; Gentry, L R; Lovejoy; J C; Owen, K Q. Influence of dietary carnitine in growing sheep fed diets containing non-protein nitrogen. Small Ruminant Research (2001) 40, 13-28.

\section{EXTENT OF PREPARTUM SERUM RETINOL CONCENTRATION DECLINE AFFECTS POSTPARTUM MORBIDITY IN HOLSTEIN COWS}

\author{
Thomas H. Herdt
}

\author{
Michigan State University East Lansing, MI USA 48823
}

Serum retinol concentrations are known to decline prepartum in cows. The cause of this decline is unknown, although it does not appear to be associated with vitamin A intake. The importance of the peripartum decline in serum vitamin A to disease resistance is also unknown. The objective of this project was to determine in cows receiving a diet of adequate vitamin A concentration, the association of prepartum serum retinol concentration to the incidence of postpartum disease. In addition, the relationship of serum retinol concentration to the serum concentration of metabolites indicative of metabolic status was also determined.

One hundred-nine Holstein cows in a commercial dairy were studied. Blood samples were collected weekly starting three weeks prior to expected calving with the last sample collected in the week after calving. All samples were collected between June and August of 1999. All animals were receiving the same, totally mixed ration with dietary vitamin A concentration of 60,000 IU/kg dry matter. The first sample, the prepartum sample nearest to calving, and the postpartum sample were analyzed for serum concentrations of retinol, non-esterified fatty acids, beta-hydroxybutyrate, albumin, and cholesterol. 
The mean collection times relative to calving for each analyzed sample were $-15,-5.4$, and $3.1 \mathrm{~d}$. Mean serum retinol concentrations declined significantly $(\mathrm{p}<.05)$ from each sample to the next, with mean (and range) values of 225 (113-397), 181 (67-333), and 118 (296-39) nG/mL, respectively. Serum concentrations of NEFA increased $(\mathrm{p}<.05)$ from each sampling time to the next. Serum retinol and NEFA concentrations were correlated negatively $(\mathrm{p}<.01)$ at each sampling time with respective $r$ values of $-.32,-.53$, and -.42 . Weaker, but significant $(\mathrm{p}<.05)$ correlations were observed between serum retinol and serum beta-hydroxybutyric acid at the immediate pre-, and postpartum samplings. Other serum metabolite concentrations were not significantly correlated with serum retinol.

Overall morbidity among the study animals was $48 \%$ with the majority due to metritis $(33 \%)$ and mastitis (16\%). The association of metabolite concentrations with disease was assessed by logistic regression. Initial independent variables in the model included twining, parity, sex of calf, calving difficulty, and the individual metabolite concentrations. Low serum retinol concentration at the sampling immediately prior to calving was a significant risk factor for overall postpartum morbidity $(\mathrm{p}<.05)$. No other independent variable entered the model.

I conclude that peripartum serum retinol concentrations are highly variable among animals, even on the same diet. Serum retinol concentration appears to be affected by general metabolic status, especially by energy status. The negative association between prepartum retinol concentration and postpartum disease might be due to insufficient delivery of vitamin A to tissues; however, low serum retinol may be general sign of metabolic stress and thus associated only indirectly with disease risk.

\title{
IMMUNOGENOMICS APPROACHES TO UNDERSTANDING PERIPARTURIENT MASTITIS SUSCEPTIBILITY IN DAIRY COWS
}

\author{
Jeanne L. Burton, P.S. D. Weber, J.B. Wells, Sally A. Madsen, and P.M. Coussens \\ Immunogenetics Laboratory and the Center for Animal Functional Genomics, 1205E Anthony Hall, \\ Department of Animal Science, Michigan State University, East Lansing, Michigan 48824
}

Studies comparing in vitro functional capacities of leukocytes from non-parturient and periparturient dairy cows have provided substantial evidence that systemic and local mammary immune defenses are deficient around parturition. This evidence has lead to the reasonable hypothesis that immune deficiency underlies the heightened mastitis susceptibility of periparturient cows. Nutrition and vaccine studies partially substantiate this hypothesis, showing that dietary antioxidant supplementation and rigorous immunization regimes can bolster innate and humoral immunity to the point that mastitis severity and time for return to normal milk production are reduced. However, completely effective resolution of this significant production disease has not been achieved because so little is understood about its complex etiology. In particular, we possess almost no knowledge of how or why immune cells responding to parturient physiology end up with deficient functional capacities. Therefore, a thorough understanding of leukocyte biology in periparturient dairy cows would seem 
a critical goal for future development of effective mastitis prevention strategies. Recently, our group has used a combination of functional genomics techniques to interrogate leukocyte RNA for global gene expression changes occurring around parturition. Leukocyte RNA was obtained for this work at multiple times before, during, and after parturition. Preliminary screening for differentially expressed genes using DDRT-PCR, dot blot, and slot blot analyses revealed profound reductions in neutrophil expression of 13 transcripts putatively identified by cDNA sequence and BLAST analyses as genes required for cellular adhesion, energy metabolism, and gene transcription. This is problematic because mammary defense against mastitis-causing bacteria depends on adequate activation, migration, and phagocytic and killing capacities of blood-derived neutrophils. Further interrogation of the leukocyte RNA using cDNA microarrays spotted with $>700$ unique genes from our group's bovine total leukocyte cDNA library revealed $~ 30$ additional genes with 3-fold or higher expression changes at parturition. Top BLAST results identified these genes as members of nuclear transcription factors, growth and differentiation factors, enzymes and cofactors of energy metabolism, antigen presentation molecules, cytokine receptors, adhesion molecules, coagulation factors, and protein kinases. Preliminary analyses also showed that many of the down-regulated neutrophil genes correlated strongly with massive fluctuations in blood concentrations of cortisol, progesterone, and estrogen. These potent steroid hormones have well-established gene regulatory actions in other cell systems, so it was not surprising that they also associated with altered gene expression in neutrophils. Chronic shifts in key nutrient partitioning hormones and nutrients in periparturient cows may also affect the expression of critical leukocyte genes, perhaps even up to the time of peak lactation. We plan to test these hypotheses in future studies. Once the most critical candidate genes of periparturient immune deficiency have been identified, these can be tested for polymorphism and as potential regulatory targets to improve mastitis resistance through novel genetic selection, nutritional, pharmacological strategies.

\title{
FUNCTIONAL GENOMICS TO STUDY COMPLEX DISEASES IN LIVESTOCK SPECIES
}

\author{
Paul M. Coussens, Jianbo Yao, Susan Sipkovsky, William Nobis, Matthew Coussens, and \\ Jeanne L. Burton
}

Center for Animal Functional Genomics, Department of Animal Science, Michigan State University East Lansing, MI 48824

\begin{abstract}
Many diseases in production animals display a complex interplay between host immune responses and pathogen-specific events. Indeed, many of the pathologies of diseases such as Johne's disease and mastitis result from the host immune response or immunopathology as opposed to direct effects of the pathogen. In addition, numerous factors may affect the outcome of any particular infection event. Production stresses such as heat, transport, parturition and nutrition all combine to dramatically alter the immune status of the host. With such complex interactions, it is clear that single gene approaches in highly controlled environments cannot describe or predict the outcome of most pro-
\end{abstract}


duction diseases. The Center for Animal Functional Genomics at Michigan State University was created to address this shortfall by applying global gene expression profiling techniques to complex problems in livestock and companion animals. Our working hypothesis is that global gene expression profiling will divulge clues to the basic biological mechanisms underlying host immune responses and interactions with various pathogens. As a first step in testing this hypothesis, we have created a high quality normalized cDNA library from total bovine leukocytes (BOTL) and used this library to produce cDNA microarrays. The first of these arrays contained approximately 720 unique genes and appropriate controls. Subsequently, bovine sequences representing over 450 key genes encoding various chemokines, cytokines, chemotactic factors, adhesion molecules, transcription factors and other regulatory factors have been obtained. A batch primer tool was used to derive primer sequences to these genes and amplicons of the various factors added to the BOTL cDNA microarrays. In initial studies using a predictable model of immune activation, stimulation of peripheral blood mononuclear cells with concanavlin A (ConA) resulted in dramatic up regulation of over 85 genes. Interestingly no genes were down regulated following stimulation with ConA. Preliminary clustering of these genes by product function shows that many of the stimulated genes encode transcription factors, adhesion molecules and proteins involved in signal transduction or cell cycle regulation. We have begun to apply our BOTL cDNA microarrays to samples obtained from animals undergoing various forms of production stress, such as transport and parturition. In addition, these arrays have been combined with differential display reverse transcriptase PCR (DDRT-PCR) in an attempt to more fully understand the complex interactions of Mycobacteria with bovine macrophages. Our results to date suggest that various forms of production stress may result in altered immune function by hampering various signal transduction pathways and reducing expression of key factors involved in maintaining immune cell viability. We have also identified at least one gene that is normally upregulated following phagocytosis, but appears to be preferentially inhibited following uptake of pathogenic Mycobacteria by bovine macrophages. These preliminary results clearly demonstrate that global gene expression profiling offers an outstanding opportunity for animal scientists and veterinarians to gain new insights into complex production diseases.

\title{
DEVELOPMENT OF A RAPID METHOD FOR DETECTION OF GRAM-POSITIVE AND -NEGATIVE BACTERIA IN MILK FROM COWS WITH SEVERE AND MODERATE CLINICAL MASTITIS
}

\author{
Siamak P. Yazdankhah ${ }^{1}$, Henning Sørum ${ }^{1}$, Hans Jørgen S. Larsen ${ }^{1}$, and Geir Gogstad ${ }^{2}$ \\ ${ }^{1}$ Department of Pharmacology, Microbiology and Food Hygiene, Norwegian School of Veterinary Science, \\ P. O. Box 8146 Dep, N-0033, Oslo, Norway. ${ }^{2}$ Procaryo AS, Kjelsåsveien 172, 0884, Oslo, Norway
}

A rapid method for demonstration of Gram-positive and Gram-negative bacteria in milk is described. The technique is based on dilution of the sample in a medium, followed by filtration through a porous polysulfone membrane with a pore size retaining and concentrating bacteria from the sample. The bacteria concentrated on the surface of the membrane are stained with a cationic dye (toluidine blue) 
that can be visualized by the naked eye. After staining, the membrane is treated with ethanol-acetic acid ( $\mathrm{pH}$ 2.8-3.0), which causes decolorization of Gram-negative bacteria, whereas Gram-positive bacteria retain the stain. The method does not require heat fixation, electrical power, microscopic examination or specially trained personnel. The time needed to perform the test is approximately 5 min. The technique was applied to artificially infected milk and milk from cows with severe and moderate clinical mastitis for detection and differentiation of bacteria. The sensitivity of the filtration method was $92 \%$, and $100 \%$ for Gram-positive, and Gram-negative bacteria, respectively, compared with traditional bacteriological culture of milk samples from cows with severe and moderate clinical mastitis. The detection limit was $5 \times 10^{6} \mathrm{CFU} / \mathrm{ml}$ for Staphylococcus aureus and $1 \times 10^{6} \mathrm{CFU} / \mathrm{ml}$ for Escherichia coli in spiked milk samples. The overall specificity of the method was $86 \%$. This diagnostic method can provide on-site guidance to the veterinarian to optimize use of antibiotics in mastitis therapy.

\title{
PROTOTHECA ZOPFII? MASTITIS IN HOLSTEIN DAIRY HERDS AND ITS CHARACTERISTICS OF INSUFFICIENT ELIMINATION FROM THE UDDER
}

\author{
H. Nagahata, ${ }^{1}$, K. Kawai ${ }^{2}$, H. Higuchi ${ }^{1}$, W. Miki ${ }^{2}$ and T. Kayama ${ }^{2}$ \\ ${ }^{1}$ Department of Animal Health, School of Veterinary Medicine, Rakuno Gakuen University, Hokkaido \\ 069?8501, ${ }^{2}$ Tokachi Vet Clinical Center, Obihiro, Hokkaido 080, Japan.
}

Prototheca spp cause sporadic cases of bovine mastitis as well as serious herd mastitis problems. Clinical mastitis caused by Prototheca zopfii (P.zopfii) occurred in 2 Holstein dairy herds. Epidemiological, bacteriological, pathological studies and therapeutic trials were performed to find the effective control measures for P.zopfii mastitis. Serum antibody to P.zopfii was monitored to evaluate the efficacy of drug therapy. Further, in vitro responsiveness of phagocytic cells to P.zopfii was also evaluated. P.zopfii was isolated from 46 quarters from 28 cows in 2 herds of 75 lactating cows by culturing quarter milk samples. Chemiluminescent response of bovine neutrophils against large size (13?20 um) of P.zopfii was 52\% of those of small size (6?8 um). Phagocytic activity of neutrophils to P.zopfii was 35\%. Pathological findings showed the chronic granulomatous changes of the inflamed mammary glands. Therapeutic trials using kanamycin $(600 \mathrm{mg} / \mathrm{day}, \mathrm{IMI})$ and /or cellulase (MacerozymeR 10\%, $300 \mathrm{ml} /$ day, IMI), which were proved to be effective to P.zopfii in vitro, for 5 days, were not efficacious to treat clinical P.zopfii mastitis. However, beneficial effects of Kanamycin infusion into infected glands,

for 5 days, were observed in cases of subclinical mastitis. Difference in serum antibody to P.zopfii was found in cows between clinical and subclinical mastitis. This suggests that serum P.zopfii?antibody level is a possible parameter to determine whether anti?microbial therapy is efficacious or not.

Poor responsiveness of neutrophils to P.zopfii was demonstrated. Under insufficient sensitivity to antibiotics and the lack of effective control measures for P.zopfii, proper handling of milking procedures may play a key role for prevention of the spreading of P.zopfii pathogen. 